\title{
Use of negative information in positioning and tracking algorithms
}

\author{
Michele Albano • Senka Hadzic • Jonathan Rodriguez
}

\begin{abstract}
To avoid additional hardware deployment, indoor localization systems have to be designed in such a way that they rely on existing infrastructure only. Besides the processing of measurements between nodes, localization procedure can include the information of all available environment information. In order to enhance the performance of Wi-Fi based localization systems, the innovative solution presented in this paper considers also the negative information. An indoor tracking method inspired by Kalman filtering is also proposed.
\end{abstract}

\section{Keywords}

Distance estimation, Data fusion

\section{Introduction}

The availability of positioning information is an enabler for location based services as part of context aware applications. The position, as well as the expected direction and velocity of mobile users are basic components of the users' overall context. On the other hand, position information can even improve the communications system itself. Geo-location information can serve as complementary data to estimate and predict critical parameters for improving wireless communication networks, such as setting up location dependent load balancing schemes, reducing power consumption in multi hop wireless networks or reducing the total routing overhead by means of location based routing.

Especially in indoor environments, where Global Positioning System (GPS) does not perform well because of the absence of Line of Sight transmission between satellite and receiver, it is still a challenge to design a system able to provide accurate positioning information. A mobile terminal's position shall be estimated with alternative techniques focusing on radio signals which are provided by the terrestrial radio access networks (RANs) itself. The rapid deployment of WLAN technology, especially in dense indoor environments, made it another compelling choice for localization, relying only on the existing network infrastructure. Widespread use of the IEEE 802.11 infrastructure and the ubiquity of Wi-Fi embedded devices is the most encouraging reason for radio-frequency (RF) based indoor localization. Thus there is no need for dedicated devices for positioning applications, or alterations to existing mobile terminals.

In this paper, we propose a novel solution to increase the localization and tracking performance. Basically, this is achieved by incorporating information about nodes that are not in range, which allows us to eliminate candidate solutions. The localization system reports the location of a mobile terminal via a map of possible locations, and the map resulted by a localization round is used, after a dispersal representing the user mobility, as the input for the successive localization round. Finally, the papers proposes a metric to evaluate the trade-off between a correct location of the mobile terminal, and the size of the set of the possible locations, i.e. correctness vs precision. 
Fig. 1 Two steps in a two-stage positioning system algorithm

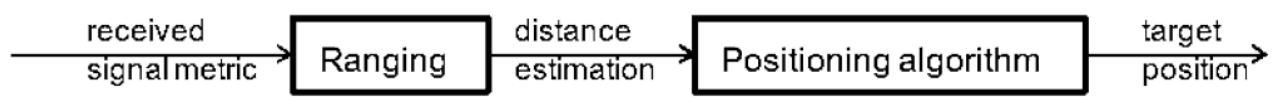

The rest of the paper is organized as follows: the next section gives an overview on related work, in Sect. 3 we describe our model and the procedures of collecting measurements and data fitting, in Sect. 4 we describe our experiments, and finally Sect. 5 concludes the paper.

\section{Related work}

Generally, the localization process assumes a number of location aware nodes, called anchors. In a typical two-stage positioning system (see Fig. 1), the first phase is the ranging phase, where nodes estimate the distances to their neighbors by observing time of arrival, received signal strength or some other distance dependent signal metric. In the second phase, nodes use the ranging information and the known anchor position for calculation of their coordinates. Unknown nodes estimate their distances to anchor nodes based on measurements of received signal strength (RSS), time of arrival (ToA) or angle of arrival (AoA). Since the RSS value is available in any IEEE 802.11 interface, it is the most appropriate measurement to use, although sophisticated models are needed to translate the received power level into distance. Once a sufficient number of distance estimates to anchor nodes is available (for $n$-dimensional space, 41 distance estimates are required), the node can compute its position using least squares algorithm.

Having in mind the limited transmission range and obstacles in an indoor environment, it is very unlikely that a mobile terminal will be able to communicate with sufficient number of anchors. For this reason, indoor localization has been a motivating research topic and many methods have been proposed so far, including Wi-Fi, RFID and UWB localization. Indoor localization techniques can be classified into two main groups: (1) The first group uses dedicated infrastructure for positioning; in this case dedicated devices have to be installed, and (2) the other group employs previously available wireless communication infrastructures. The latter group is a cost efficient solution with large coverage, while high accuracy, availability and reliability can be attained. On the other hand, there is a need for more intelligent algorithms to compensate for the low performance of measurement techniques.

Most Wi-Fi-based location approaches correspond to radio maps (fingerprinting). Although high accuracy is attainable, a complex training process is required to develop the fingerprinting database, specifically each time the environment changes.

The Active Badge System was an early system developed to localize mobile devices within a building [1]. Every badge identifies itself periodically, sending unique infrared signals to the receivers. Although it provides accurate location, the drawbacks of the system are poor scalability due to limited range of IR, and deployment cost. The system RADAR [2], based on WiFi fingerprinting, uses signal strength information from multiple receiver locations. The main idea is to record radio signals and build models for the signal propagation during off-line analysis. However the system's main disadvantage is its dependence on empirical data. The work in [3] uses neural networks based on Bayesian Regularization or Gradient Descent to obtain the location of mobile nodes from RSSI and LQI. PlaceLab [4] uses connectivity from GSM base stations and 802.11 access points. If the node density is high enough, the system achieves accuracy of 15-20 meters, which is even lower than GPS, but unlike GPS it is capable to perform localization for both indoor and outdoor environments. Both passive and active RFID devices have been considered in [5] to provide connectivity based localization. These algorithms use signal parameters such as RSS to create a radio map of the environment. Afterwards, location is estimated by matching online measurements with the existing fingerprints, collected during the offline training phase. However, the fingerprinting method is a complex procedure that requires a training process to develop the fingerprinting database, specifically each time the environment changes. Cricket [6] is a decentralized location support system for sensors based on RF and ultrasound. Incorporating ultrasound hardware was necessary because a purely RF-based system did not provide satisfactory results. It takes into account user privacy and does not depend on underlying network technology. Still, the system's granularity is a portion of a room. Other localization techniques for wireless sensor networks are described in [7].

Cooperative positioning algorithms are widely used in indoor scenarios where a line of sight connection to anchor nodes is not always available, due to short communication range, obstacles and a harsh environment. For sparse sensor networks the most widely used method is multi-dimensional scaling (MDS), a statistical dimensionality reduction technique that uses pair-wise distance measurements as input data $[8,9]$. Similarly, pair-wise distance measurements are used as convex constraints [10], and linear and semi-definite programming (SDP) techniques [11] are used to estimate locations of free nodes.

In distributed algorithms each node in the network is responsible for computing its own position. Therefore they are suitable for ad hoc networks, having in mind the nature of these networks, specifically the limited resources such as energy constraints, processing capacity and short transmission range. 
One broadly used approach is the iterative multilateration scheme, where unknown nodes, once being localized, serve as virtual anchors for the rest of unknown nodes in subsequent iterations [12-14]; one major drawback of this method is error propagation, resulting from using erroneous virtual anchors. Moreover, in [13] the authors take into account the channel behavior to provide accurate indoor positioning and importantly reduce error propagation. In [14] the authors develop an error control mechanism based on characterization of node uncertainties.

While the presented algorithms are deterministic, e.g., their aim is to find the deterministic location; statistical/ probabilistic approach is proposing an approximate solution. Generally, the aim is to estimate the maximum a posteriori node location using a set of observations (distance estimates) and a priori probability distributions of nodes' locations. The belief propagation algorithm is a graphical model for distributed statistical inference, widely used for positioning. The amount of computation is proportional to the number of links in the graph. Non parametric belief propagation is more acceptable for localization in wireless networks, because of its ability to accommodate non-Gaussian distance estimation errors and provide an estimate of the remaining uncertainty in each node location $[15,16]$. The main drawback of statistical models is that convergence is not guaranteed in networks with loops [17].

Factor graphs present another distributed solution, particularly suitable for sensor networks. In [18] the authors developed an algorithm based on estimation theory and statistical inference. The factor graphs were mapped onto the timevarying network topology, resulting in a network messagepassing scheme. The underlying technology is UWB, and measurements of interest are ToA estimates. A measurement campaign was performed to establish the appropriate ranging model.

Particle filtering methods weigh the particles according to their likelihood. Monte Carlo estimation methods are mainly used for robot localization and tracking [19]. In principle, the procedure is divided into a prediction phase, where the robot moves and its position's uncertainty increases, and an update phase where new observations are integrated to filter and update data. Anyway, the constraints in wireless sensors and ranging accuracy make node localization more difficult than robot localization [20].

Negative information had few applications for localization in wireless networks. Most of the work targeted problems for mobile robot localization [21-23]. In Markov localization for mobile robots, the absence of an expected measurement can be used to improve localization. One difficulty in implementing a system that uses negative information is that there are two main reasons for the lack of an expected measurement reading: the target may not be there or the sensor may not be able to detect the target. To avoid false negatives, the model needs to consider possible obstructions [21]. Nevertheless, even a false attempt to detect a target can be exploited in tracking applications, based on Bayesian approach to target tracking [22]. Negative information can be integrated by generating an artificial measurement. The precondition for processing negative information is a refined sensor model. However, all these works only consider cases where an expected observation is missing. In [23] the authors have shown how negative information can be incorporated into FastSLAM, a system that is alternative to the complex Extended Kalman Filter approach for robot localization. In wireless sensor localization, Monte-Carlo localization algorithms make use of negative information [24]. However, it can be useful only in obstacle-free areas, and leads to localization errors otherwise.

The requirements on localization systems are even stricter when nodes are mobile, since for real time tracking there is a demand not mainly on accuracy, but also on latency and complexity. Kalman filtering is the most widely adopted technique for location tracking. One Kalman filter based solution making use of RSS measurements has been presented in [25]. Basically, the Kalman Filter (KF) offers an optimal solution for linear systems, where measurement errors follow a Gaussian distribution. Otherwise, the KF has to be transformed into the Extended Kalman Filter (EKF), suitable for non-linear systems [26]. The EKF consists of three phases:

1. the predict phase, which computes the a priori estimation based on the previous estimate, and

2. the update phase, that occurs when a new measurement becomes available;

3. finally, the a posteriori estimate is computed.

The current location of the node depends on the previous location, which is modeled by the state transition function; in localization problems this is basically the mobility (velocity) model.

\section{Proposed technique}

\subsection{Composing different sources of (negative) information}

This subsection proposes a technique to fuse different types of information to perform localization of a unit. The technique described is as abstract as possible, since it aims only at showing the general idea. Section 3.2 will refine the technique towards the implementation in a simple wireless scenario, and Sect. 4 will provide information about the implemented system.

We propose a model where localization procedure makes use of different sources of information, that can comprise sources of negative information. In this respect, positive information means that some data is saying "you can be here", 
while data bearing negative information is saying "you can not be here". The main idea behind the system is to provide a framework to compose different kinds of information that can contribute to the localization process. Instead of applying only positive reasoning, an alternative way is to consider all the locations in the area, and provide a technique to evaluate how "unlikely" a mobile unit to be located in a given position. Our approach to localization is based on the fact that "when you have eliminated the impossible, whatever remains, however improbable, must be the truth" [27]. In fact the proposed system exploits all the available information to all possible mobile locations, resulting in a normalized probability map of probable locations.

For each possible location on the probability map, the predicted measurement is computed, and then the predicted noise is applied to it, to get a probability distribution function for the measurement. The location on the probability maps is described by its coordinates $(x, y)$ in the plane, the error $e$ we would need to match the prediction with the measurement is:

$$
e=V_{x, y}-m
$$

where $V_{x, y}$ is the predicted signal in $(x, y)$ and $m$ is the measurement. Using the symbol $F$ for the pdf of the error, and $p$ for the pdf for the localization, $p$ is function of the required measurement error $e$, and is parametric in $(x, y)$ :

$$
p_{x, y}(m)=F_{x, y}(e)=F_{x, y}\left(V_{x, y}-m\right)
$$

The composition of different types of information is done by considering all the measurements with their own error, and by considering these errors as independent. Given a measurement $m_{1}$ taken from a source of information, for example the RSS from an access point, the probability for a unit to be in a given location $(x, y)$ depends on the expected measurement $\mu_{1}(x, y)$, the expected error of the signal $\sigma_{1}(x, y)$, and the predicted distribution of the signal at the location $(x, y)$. With our notation, $p_{1}$ is the probability for a measurement to be $m_{1}$. Since we are considering independent information sources, if the probability to be in the same location $(x, y)$ given a measurement $m_{2}$ from a different information source is $p_{2}$, the probability for that location is $p_{1} p_{2}$.

Now, for all possible mobile positions, we apply the same kind of reasoning to check the "compatibility" of each measurement with the expected signal. If at a given stage of the computation, the probability map is $M(x, y)$, after a given measurement is applied, the probability map is modified to $M^{\dagger}(x, y)$ :

$$
M^{\prime}(x, y)=M(x, y) p_{x, y}(m)=M(x, y) F_{x, y}\left(V_{x, y}-m\right) \quad(3)
$$

If we start from a probability map where all the probabilities are, for example, equal to 1 , we will end up with a map where a number of locations are ruled out, while a set of locations are still quite probable. Now we apply the normalization process, where all the probabilities are multiplied by the same number such that the maximum value in the probability map is 1 . On the other hand, if we consider that we have already localized the mobile terminal, a special source of information that we can apply to the probability map is the history of the mobile terminal location. The map obtained in the previous localization step is used as input for the following localization round, after applying a dispersal algorithm to represent the user mobility.

In principle, an approach could first display the probability distribution of a node's position based on signal strength measurements from all access points that are in range. Afterwards, we update this distribution by incorporating negative information: if a signal measurement is missing, we consider it as a signal that is too weak to be received, and we set its value as some conventional value. The fact that a node is not able to sense certain access points gives us the possibility to update the probability distribution, by ruling out some potential solutions to the localization problem.

It is possible to apply a threshold $\tau$ to the probability map, to consider that the mobile unit can be in all the locations where the probability value is higher than $\tau$, while it can't be in the locations where the probability is lower than $\tau$. The threshold is useful both for visualization purposes, and as a metric for the localization:

- From the point of view of the visualization, the threshold is used to show the final user a map with only the locations where the user is likely to be located.

- As a metric, while tuning up the technique, it is possible to consider the size of the "feasible locations" map, and the distance between this area and the real location of the user. The more stringent the threshold, the smaller the "feasible location" area, but the more probable that the real user location will fall out of the "feasible location" area. Hence, it is possible to experiment with the threshold to evaluate the trade-off between a correct location of the mobile terminal, and the size of the set of the possible locations, i.e. correctness vs precision.

The proposed technique is able to provide two main benefits:

- composition of information from multiple sources: every source of information is considered with its error and its distribution, to evaluate the compatibility of the measurement with a given location $(x, y)$. Moreover, the probability of location $(x, y)$ is just the multiplication of all the probabilities that are extracted from the single measurements;

- exploitation of negative information: we are not giving value only to information that validates a given location. On the opposite, we consider valuable all the information, for example the absence of the RSS from an access 
point. In this case, the system would estimate the probability for the signal to be low enough not to be received, and would exploit that probability for generating the probability maps.

\subsection{Implementing the proposed technique}

This subsection proposes the design of a prototype for the localization system. The scenario that we consider is a wireless scenario, where a mobile unit (e.g.: a laptop) is in range with a number of IEEE 802.11 access points.

When a node is sensing available access points, some of them can be detected and the others not. Our information is increased by knowing the fact that some of the access points could not be sensed. The measurements of interest are the RSS values, since these are readily available in IEEE 802.11 interfaces. During the scanning phase, a node performs sensing to identify all the available access points.

We limit the system to using a simple lognormal signal model [28] to translate the RSS values to distances, and hence to probabilities for given locations. We are aware of the limitations of this model in terms of predicting power for the RSS, but we chose it on purpose to test our proposed technique against poor signal processing techniques. If the system will be able to perform reasonably, we can conclude that applying refined signal processing techniques and a more reasonable signal propagation model, such as the ones described in [29] and [30], would further improve the localization performance.

When the user localization performed in a given round, is used as a basis for a new localization, a dispersal algorithm is applied to the map. In particular, it is considered that the user can move up to a speed $v$ measured in meters/seconds, and that the localization algorithm uses measurements taken every $\theta$ seconds. In the rest of the discussion, we use the symbol $M_{i}$ to address the localization map obtained after the application of the algorithm in round $i$, and $M_{i}^{\mathrm{t}}+$ for the map that is feed into the localization algorithm for round $i$. Both the maps are functions $M(x, y)$ from a pair of coordinates $(x, y)$ to a number that is the probability density that a terminal is located in $(x, y)$. The map $M_{i_{+1}}^{\mathrm{t}}$ is computed as follows:

1. for each location $\left(x^{\mathrm{t}}, y^{\mathrm{t}}\right)$ of the map $M_{i_{+}}^{\mathrm{t}}$, area $A_{x^{\mathrm{t}}}, y^{\mathrm{t}}$ is the set of points in $M_{i}$ that are at most at distance $v \theta$ from $\left(x^{\mathrm{t}}, y^{\mathrm{t}}\right)$, that is,

$$
\begin{aligned}
A_{x^{\prime}, y^{\prime}}= & \left\{(x, y) \in M_{x^{\prime}, y^{\prime}}^{\prime}\right. \\
& \text { with } \left.\left(x-x^{\prime}\right)^{2}+\left(y-y^{\prime}\right)^{2}<(v \theta)^{2}\right\}
\end{aligned}
$$

2. for each point $(x, y) \in A_{x^{\prime}}, y^{\prime}, M_{i}(x, y)$ is added to $M_{i+1}^{\prime}\left(x^{\prime}, y^{\prime}\right)$, that is,

$$
M_{i+1}^{\prime}\left(x^{\prime}, y^{\prime}\right)=\sum_{(x, y) \in A_{x^{\prime}, y^{\prime}}} M_{i}(x, y)
$$

3. an optional step is to perform the normalization of the map $M_{i+1}^{\mathrm{t}}$, to have either

$$
\begin{aligned}
& \max _{\left(x^{\prime}, y^{\prime}\right)} M_{i+1}^{\prime}\left(x^{\prime}, y^{\prime}\right)=1 \\
& \text { or } \\
& \sum_{\left(x^{\prime}, y^{\prime}\right)} M_{i+1}^{\prime}\left(x^{\prime}, y^{\prime}\right)=1
\end{aligned}
$$

We consider that a tuning phase has been executed in the area, with the goal of finding the parameters of the simple lognormal signal model, and we consider that for each access point, some part of the area is behaving like a Line of Sight (LoS) signal transmission, while the rest is behaving like a non Line of Sight (nLoS) signal transmission. Thus, for the prediction of the RSS of the signal, we use two functions, one for LoS distances and one for $n$ LoS distances, with $d$ the Euclidean distance between the access point and the location $(x, y)$. Both the functions are of the form:

$$
V_{x, y}=R S S_{0}-10 * n_{p} * \log \left(d / d_{0}\right)
$$

where $R S S_{0}$ is the received power at reference distance $d_{0}$ (we assume the usual value for reference distance $d_{0}=1 \mathrm{~m}$ ), $n_{p}$ is the path loss exponent. The functions for LoS and $\mathrm{nLoS}$ differ only for the values of $R S S_{0}$ and $n_{p}$, and this translates into two system-wide set of parameters for the signal propagation, one set applied to all the access points with LoS access, and the other set applied to access points with nLoS. In both cases, we consider that the error on the received signal strength has Gaussian statistics, with $5 \mathrm{dBm}$ width for the LoS signal, and $7 \mathrm{dBm}$ for the nLoS signal, as suggested in [31]. When a signal is missing, we consider it as a poor signal, and we set its RSS to the value of $70 \mathrm{dBm}$.

Although the tuning phase adds a setup time to our technique since it is necessary to perform the tuning for every single scenario, one motivation for the simple lognormal signal model is that it uses only 2 parameters to describe signal propagation, and hence a limited number of measurements can be sufficient for fitting the wireless channel parameters.

\section{Experiments}

We illustrate our model based on measurements performed on the second floor of the Instituto de Telecomunicações building (Fig. 2). The dimensions of the area are about $50 \mathrm{~m}$ by $50 \mathrm{~m}$. There are three access points in an indoor environment (represented on Fig. 2 by a small thunder). We recorded measurements from a laptop to the access points, at several locations in the building. Communications are performed by using the WLAN 802.11g standard. The speed of the mobile terminal when performing tracking is $v=1 \mathrm{~m} / \mathrm{s}$, and the measurements used to perform localization are taken every $\theta=4 \mathrm{~s}$. 


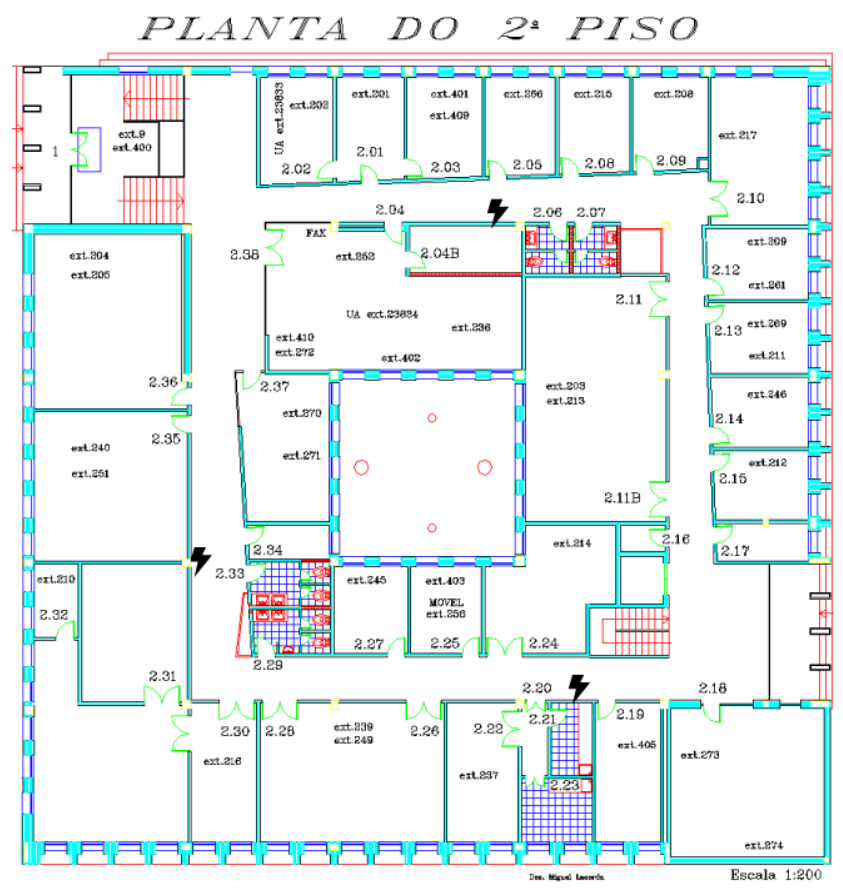

Fig. 2 Floor 2 of the Instituto de Telecomunicações, and access points' location

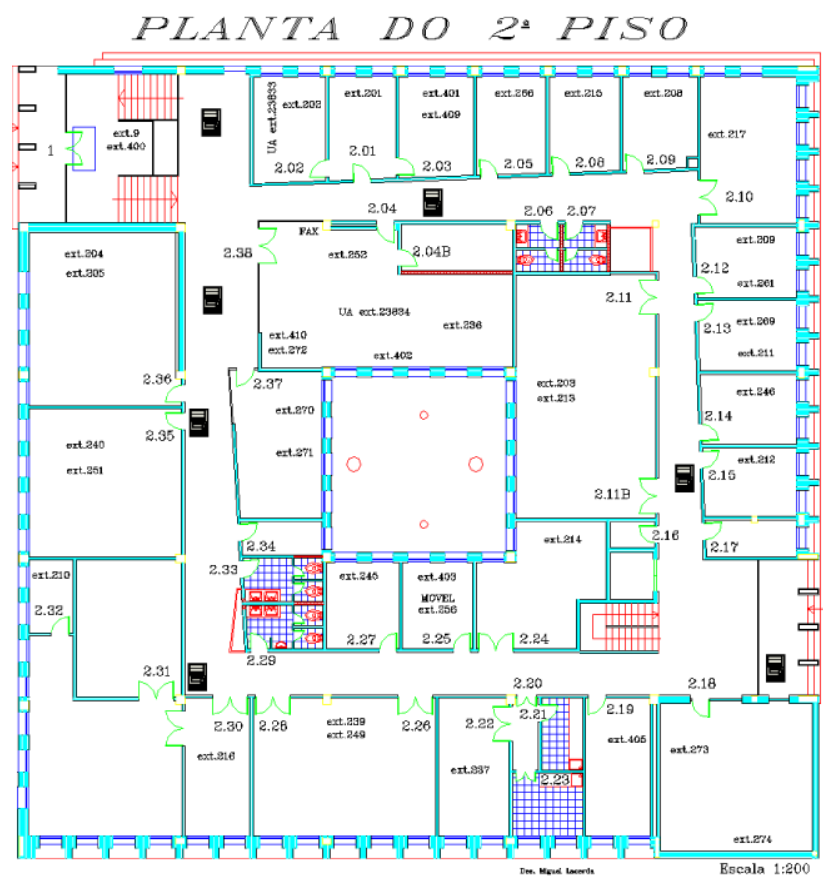

Fig. 3 Locations where data were taken for the tuning of the mobility model

\subsection{Tuning of the system}

Measurements were taken on the locations shown in Fig. 3. WiFi Hopper [32] was used as a tool to record the received signal strength at the mobile station from the infrastructure (access points). WiFi Hopper is a WLAN utility with

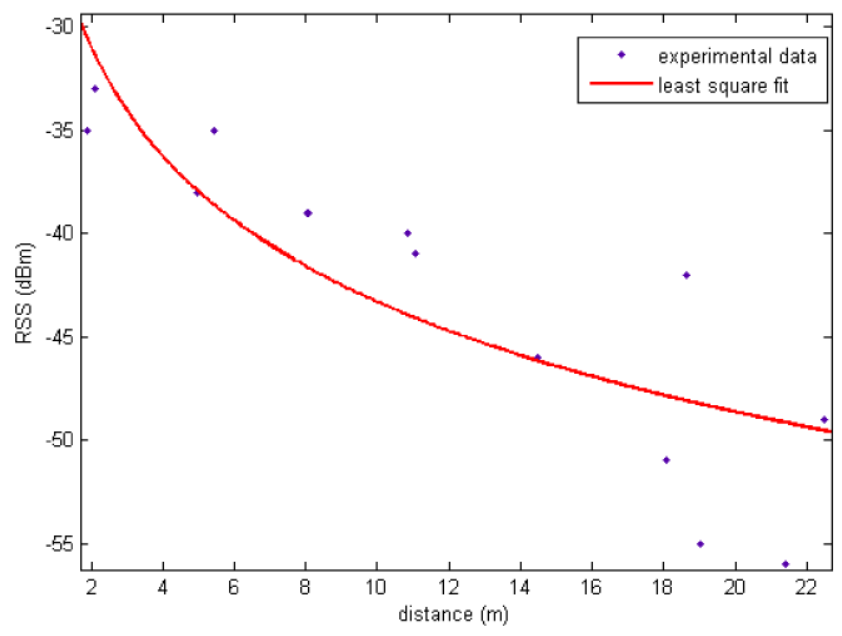

Fig. 4 Fit for the simple lognormal parameters, access points in Line of Sight

the ability to display network details like type of network, network mode (infrastructure or ad-hoc), received signal strength (RSS values), frequency and channel, encryption type etc. We performed several measurements inside the building using a Toshiba Satellite equipped with an Atheros network adapter, running Windows Vista and using the basic drivers the operating system is shipped with. RSS values from all three access points were collected, both with and without Line of Sight. As stated in Sect. 3.2, to translate RSS values into distances $d$, we use the simple lognormal model, shown in Eq. (6), where $R S S_{0}$ is the received power at reference distance $d_{0}$ (we assume the usual value for reference distance $\left.d_{0}=1 m\right), n_{p}$ is the path loss exponent.

The simplified path loss model is defined for example on p. 40 of [33] as:

$$
P_{r}(\mathrm{dBm})=P_{t}(\mathrm{dBm})+K(\mathrm{~dB})-10 n_{p} \log \frac{d}{d_{0}}
$$

In Eq. (7), $K$ is a constant which depends on the environment. When the simplified path loss model is used to approximate experimental measurements with line of sight, the value of $K$ can be set to the free space path loss at reference distance $d_{0}$. Knowing that the EIRP for the access point is $15 \mathrm{dBm}$, and the $2.4 \mathrm{GHz}$ frequency offers a path loss at reference distance $1 \mathrm{~m}$ of $39.9 \mathrm{~dB}$ (calculation based on the Free Space model), we adopt the value of $24.9 \mathrm{dBm}$ for $R S S_{0}$. As we can see from Fig. 4, for the case when measurements were taken from access points that have Line of Sight connection, the data fit returned value $n_{p}=-1.827$.

For the NLOS case we determine the constant $K$ together with $n_{p}$ by applying the least square fit to experimental data. Having in mind that the EIRP is identical as in the LOS case, we obtained the values $n_{F} \mathbf{5 . 7 6 9}$ for the path loss exponent, and $K=11.58$. The fit based on experimental data can be seen in Fig. 5. Rather small number of total measurements provided us a rough approximation of the parameters for the simple lognormal model. 


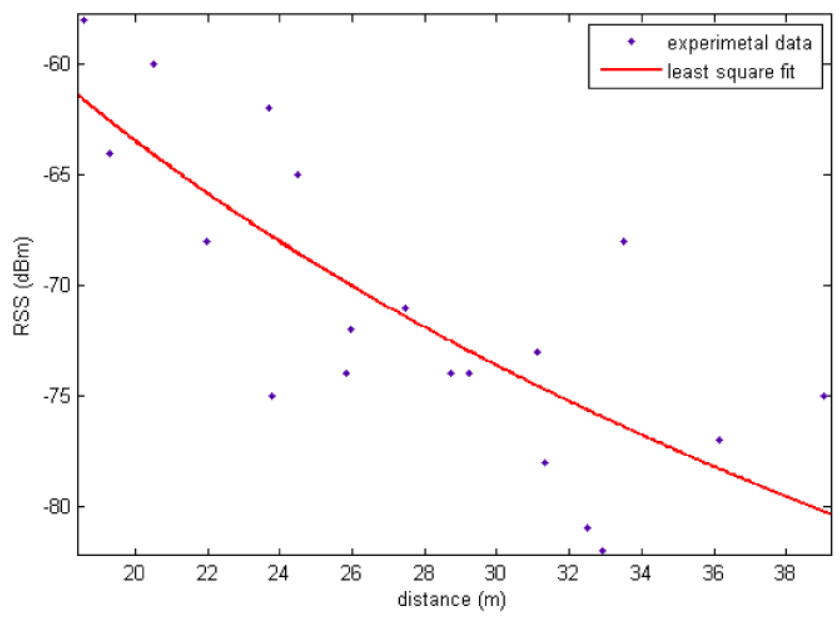

Fig. 5 Fit for the simple lognormal parameters, access points NOT in Line of Sight

However, for accurate modeling of propagation parameters, it would be essential to predict complete received signal statistics and consider the effect of variable shadowing due to the movement of people in the observed area [34]. Empirical calculation of those parameters needs to consider losses originating from obstacles of varying material, size and number [35]. In both cases, the fit that we used reported a pretty unprecise matching with the values, hence we can predict that the localization system will not provide perfect localization, but will have to exploit the composition of all available information, with the goal of providing a good localization of the mobile unit.

\subsection{Localization of the mobile unit}

The experiments involved measuring the RSS values from the three access points, computing for each measurement the pdf, and multiplying these three probability densities to find out the probability density of a given location. The visualization process was performed by applying a mask to the floor plant, where the dark areas refer to the possible mobile locations.

Figures 6, 7, 8 and 9 show the localization of the same mobile unit, represented in the figures by a small white + sign. The first three figures represent the probability maps for each of the access points (where + is the mobile unit, and the small thunder is the access point). Even though the real location of the mobile unit matched with the probability map, the localization was not precise since a number of locations featured a high compatibility with the RSS measurement. Figure 9, on the other hand, constitutes the composition of the probability map of all the access points. The result shows that the mobile unit is considered to be in a well defined area, either in the corridor, which is its actual position, or in the room nearby.

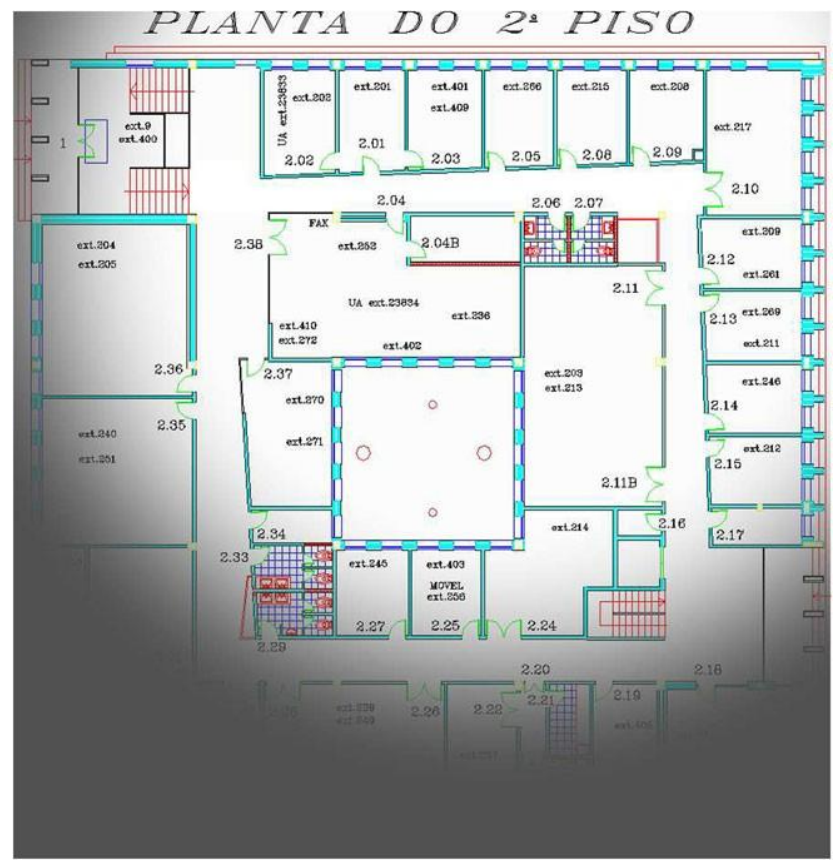

Fig. 6 Probability map when using only the first access point

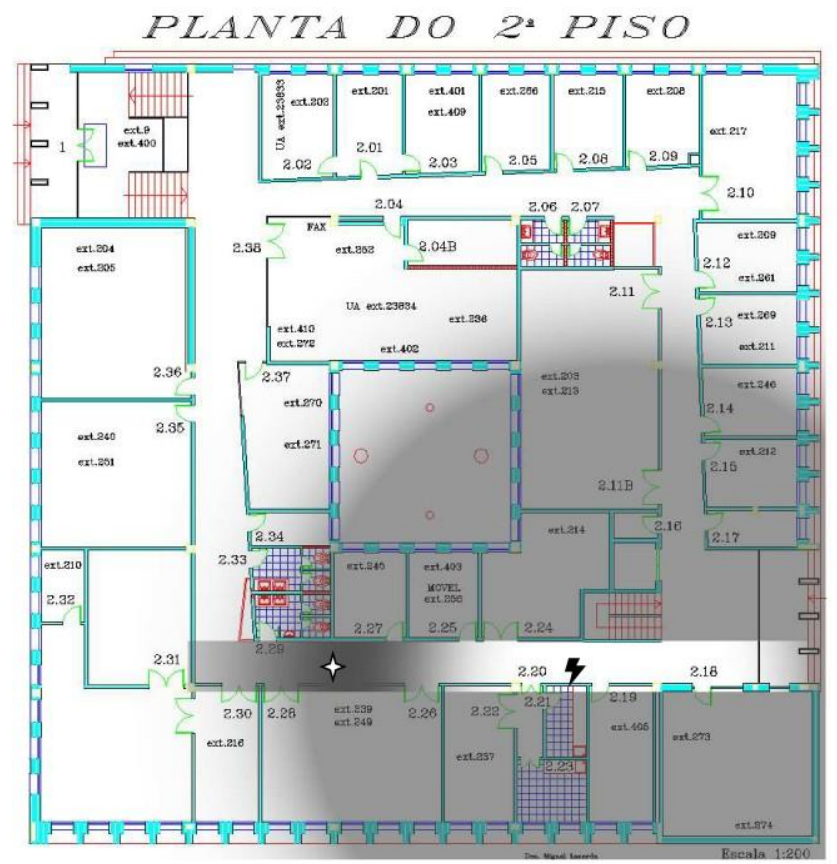

Fig. 7 Probability map when using only the second access point

Figures 10, 11, 12 and 13, show more probability maps. In each of the figures, all the three RSS measurements were used, and the real location is represented by a small white + sign. Figures 10 and 11 compare the localization results when a mobile unit moves from a location where it has just one LoS access point, to a location where it has two LoS access points. Figure 12 shows the behavior of the technique 


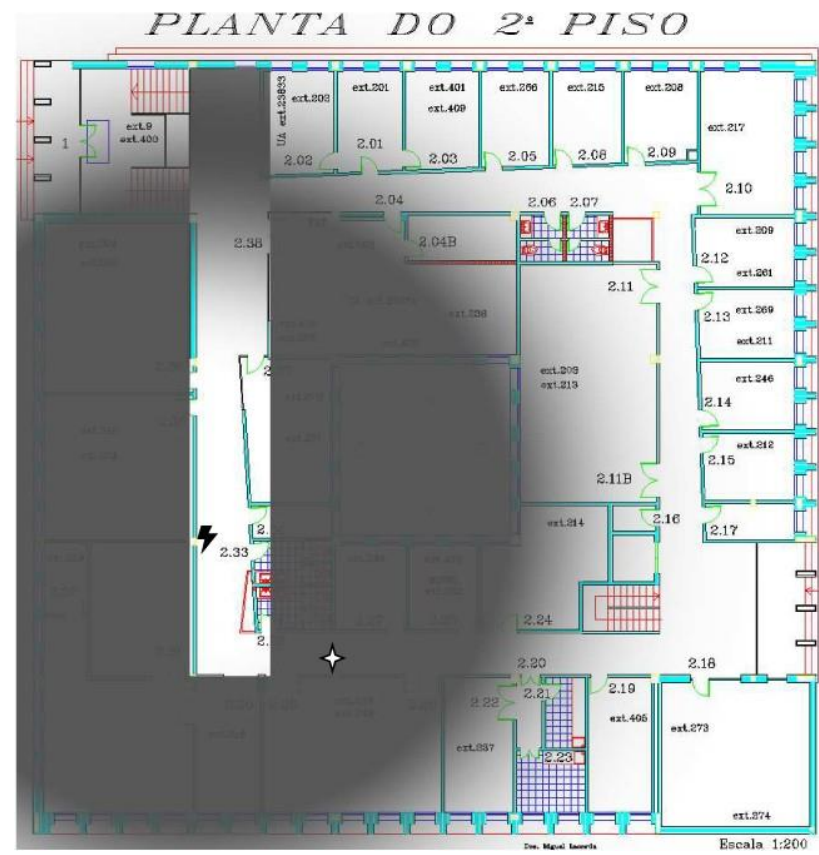

Fig. 8 Probability map when using only the third access point

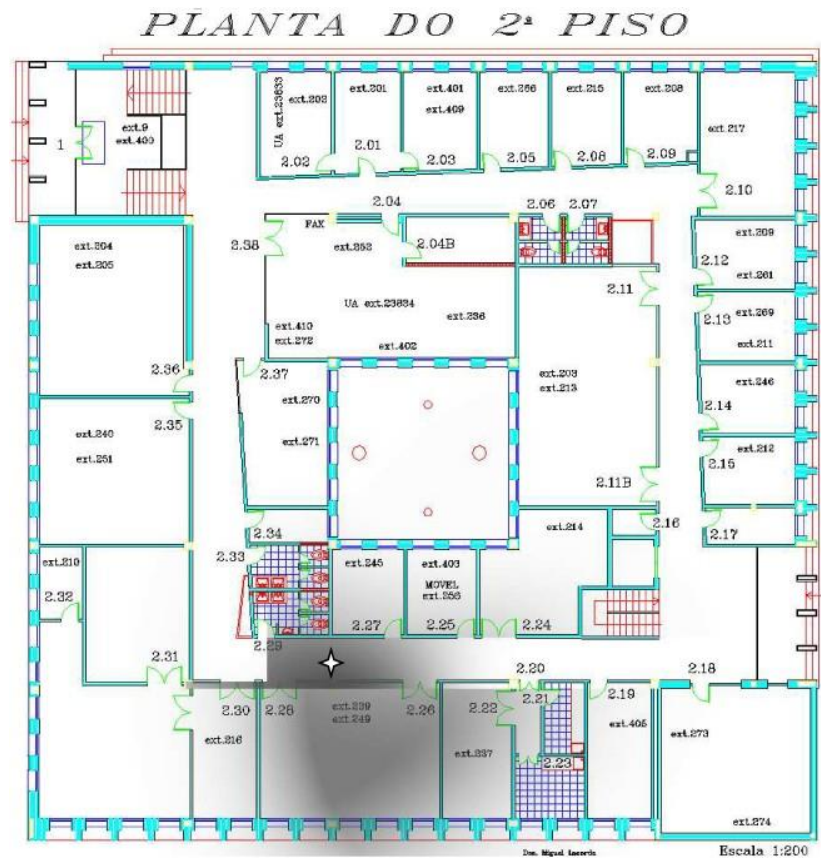

Fig. 9 Probability map when combining all available information

when there are no access points in LoS, and it confirms the limitations of the signal model we are using (simple lognormal model). Finally, Fig. 13 shows another scenario with only one LoS access point, and the localization is quite precise. We see that in some cases the method gives fairly good results. Nevertheless, we have to keep in mind that the applied channel model is very simple, serving only to illustrate the proposed scheme.

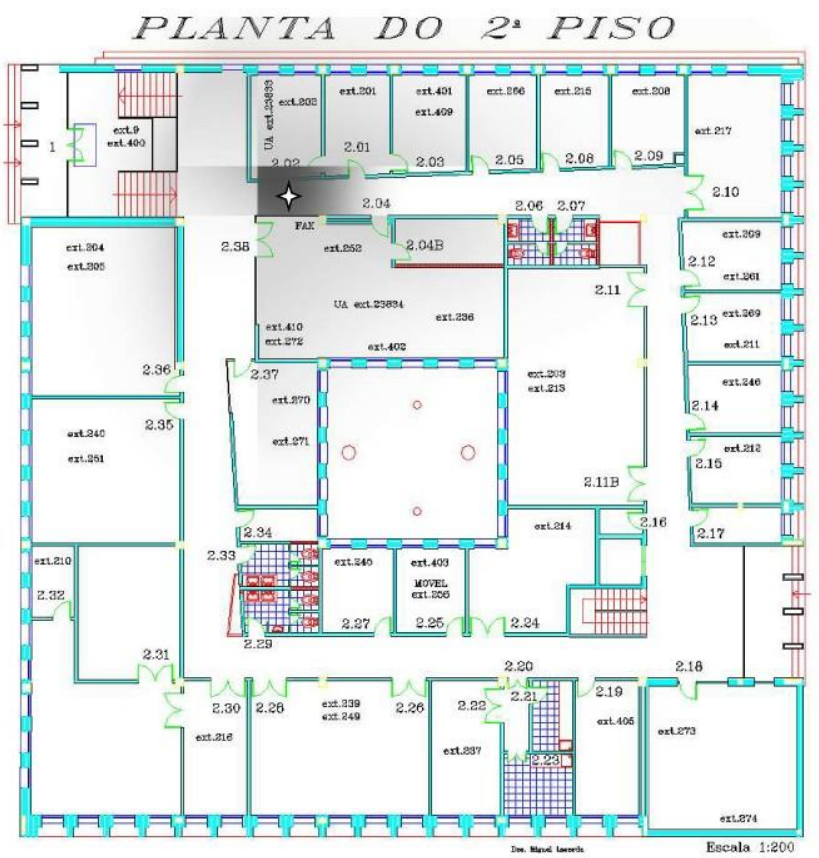

Fig. 10 Localization with one access point with LoS, two with nLoS

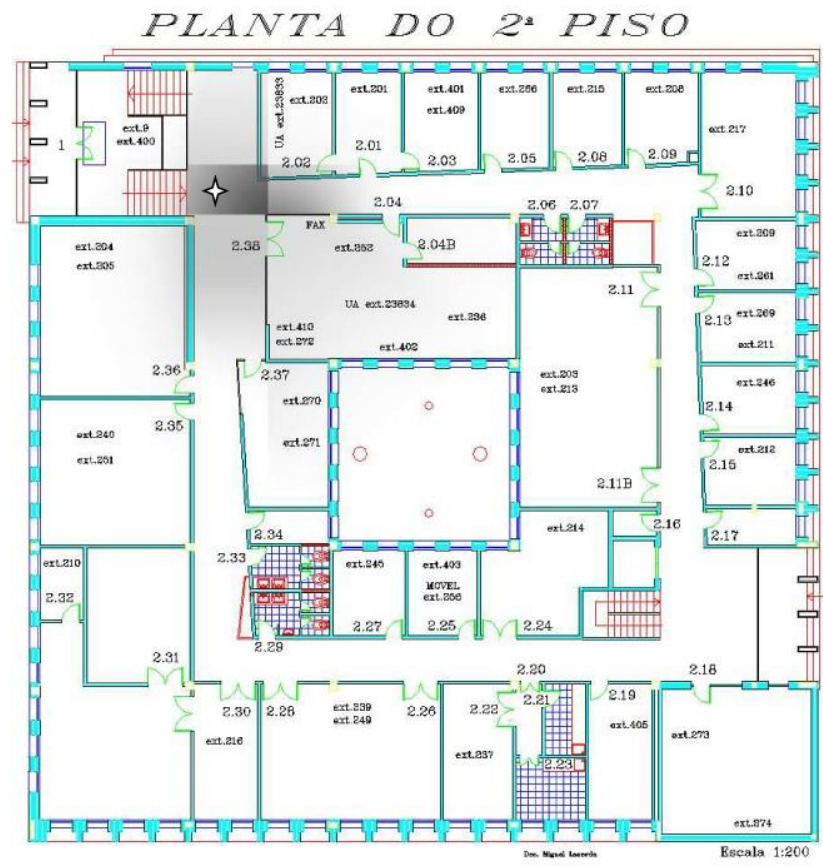

Fig. 11 Localization with two access points with LoS, one with nLoS

\subsection{Tracking by means of probability map recycling}

The experiments involving the tracking of a mobile unit involved using the posterior probability, computed at the end of a localization process, as the priori map for the successive round. Between the computation of the posteriori and its use as priori, a dispersion round is executed on the map, 


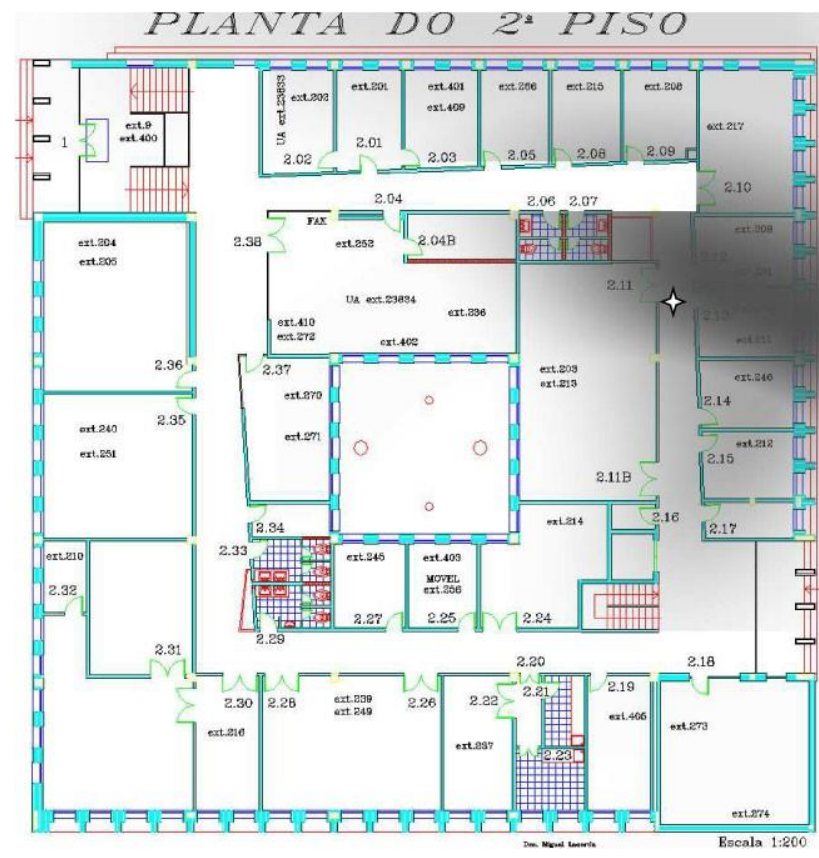

Fig. 12 Localization with three access points with nLoS

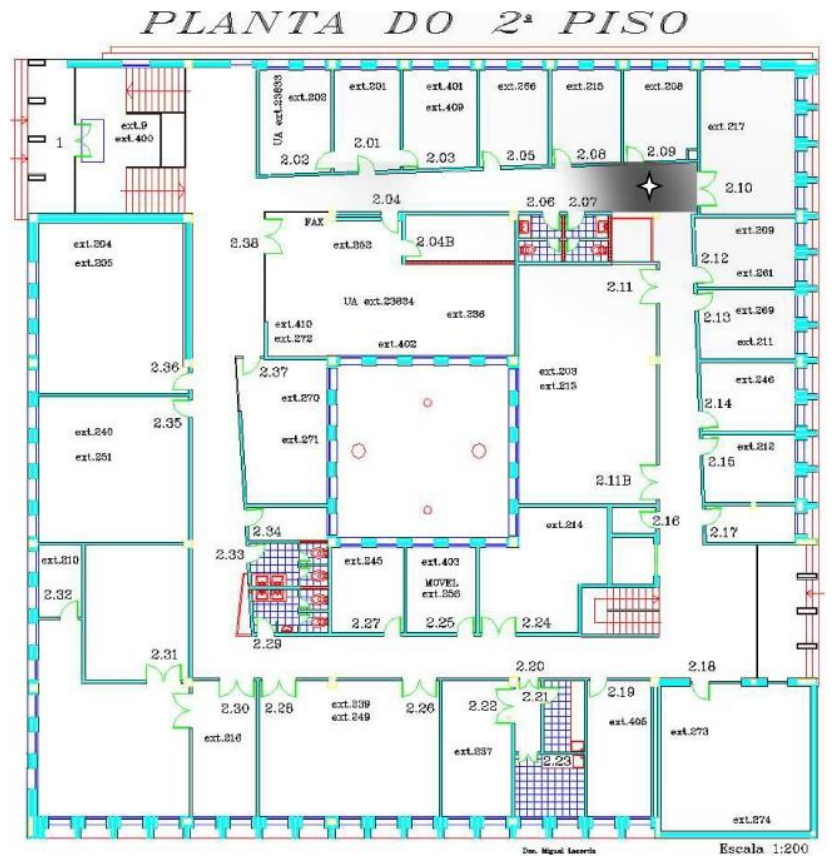

Fig. 13 Localization with one $\operatorname{LoS}$ access point, two nLoS access points

in which every location on the map adds to its own localization probability, the localization probability of all the locations in range, given a mobility model that predicts users will move at $1 \mathrm{~m} / \mathrm{s}$ and that the localization is executed once every 4 seconds.

The Figs. 14, 15, 16, 17, 18, and 19, 20, 21, 22, 23 provide a comparison of localization maps when the probabil-

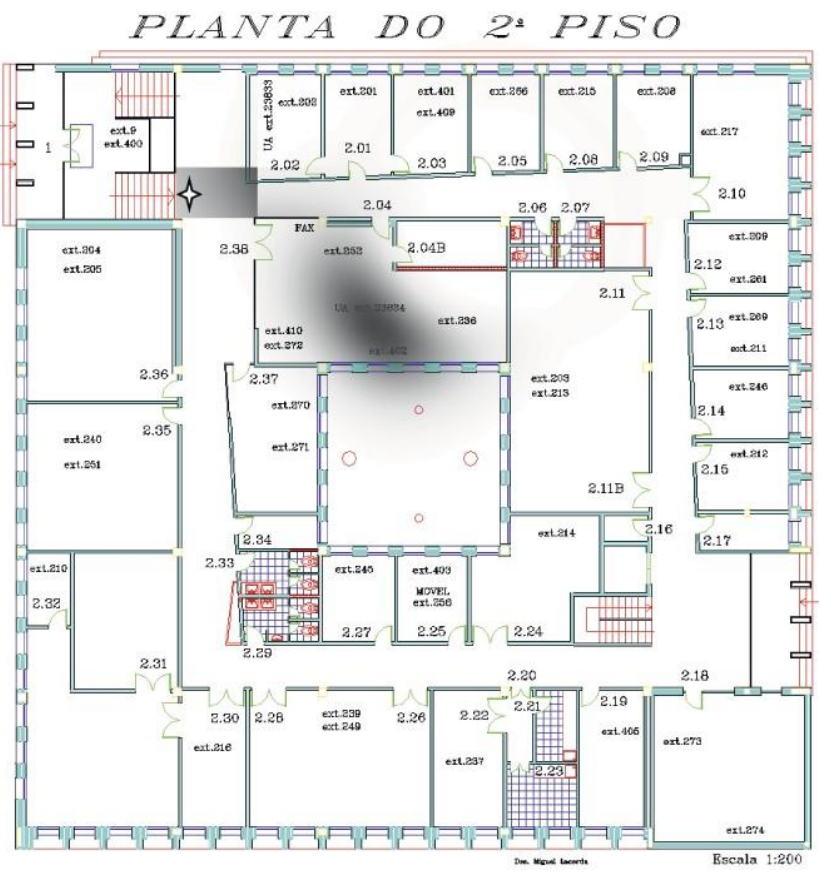

Fig. 14 Localization without recycling the old map as an "a priori"

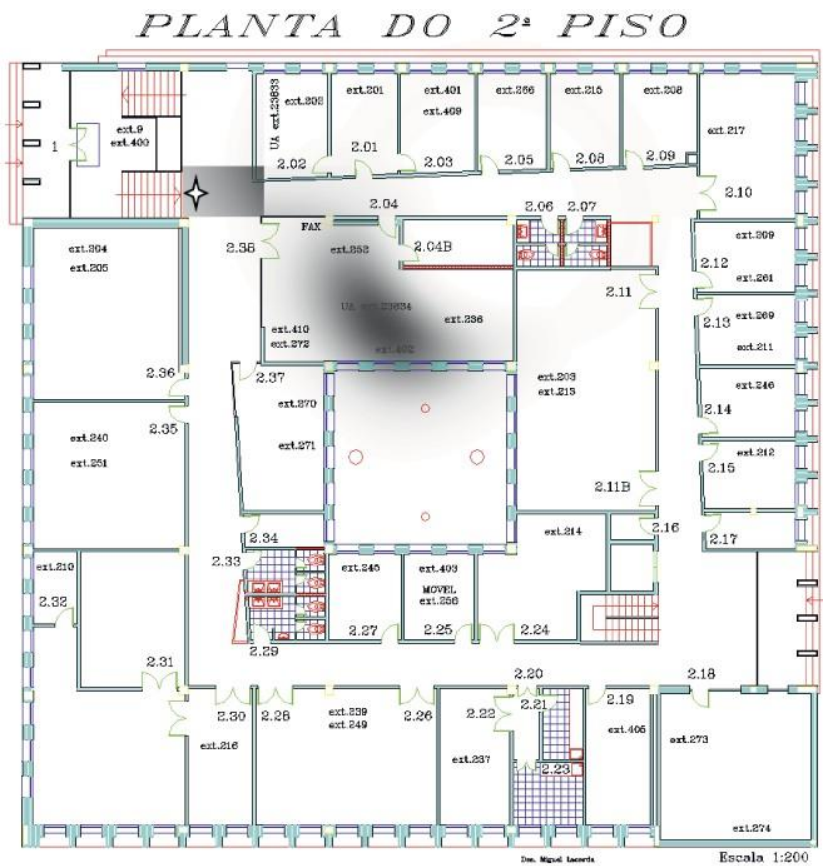

Fig. 15 Localization using the old localization map as an "a priori"

ity map is recycled, and when the previous state is ignored and localization is performed from scratch. In each figure, a small white sign shows the real location of the mobile terminal. It is clear that the system's tracking capabilities improved the system by ignoring locations that do not match with previous estimates, hence making smaller the set of potential solutions. 


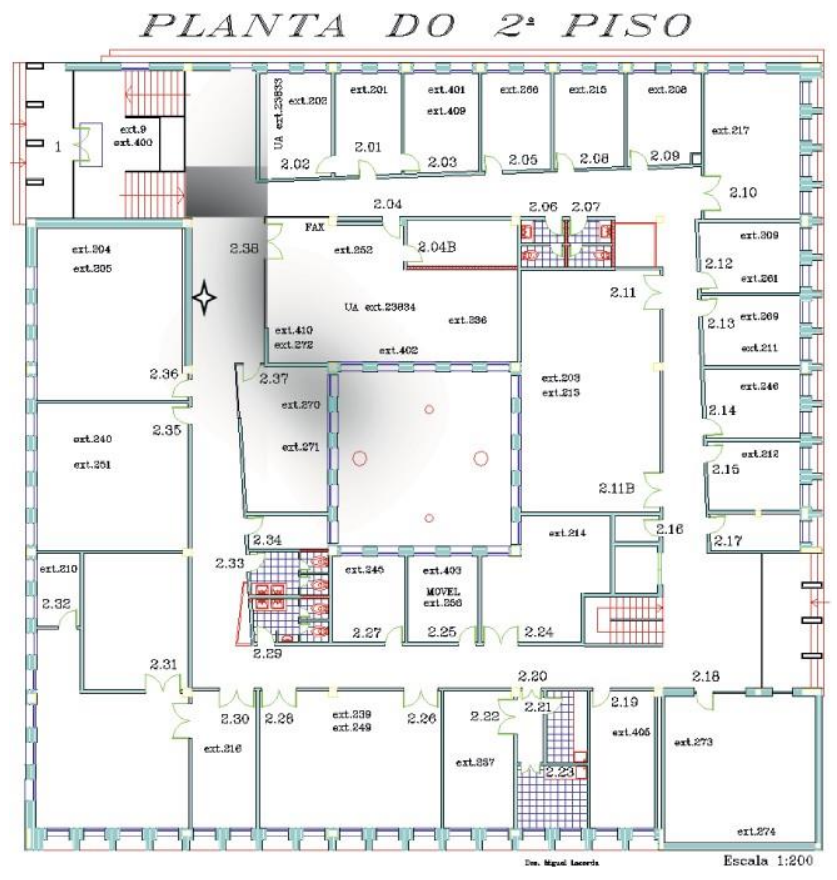

Fig. 16 Localization without recycling the old map as an "a priori"

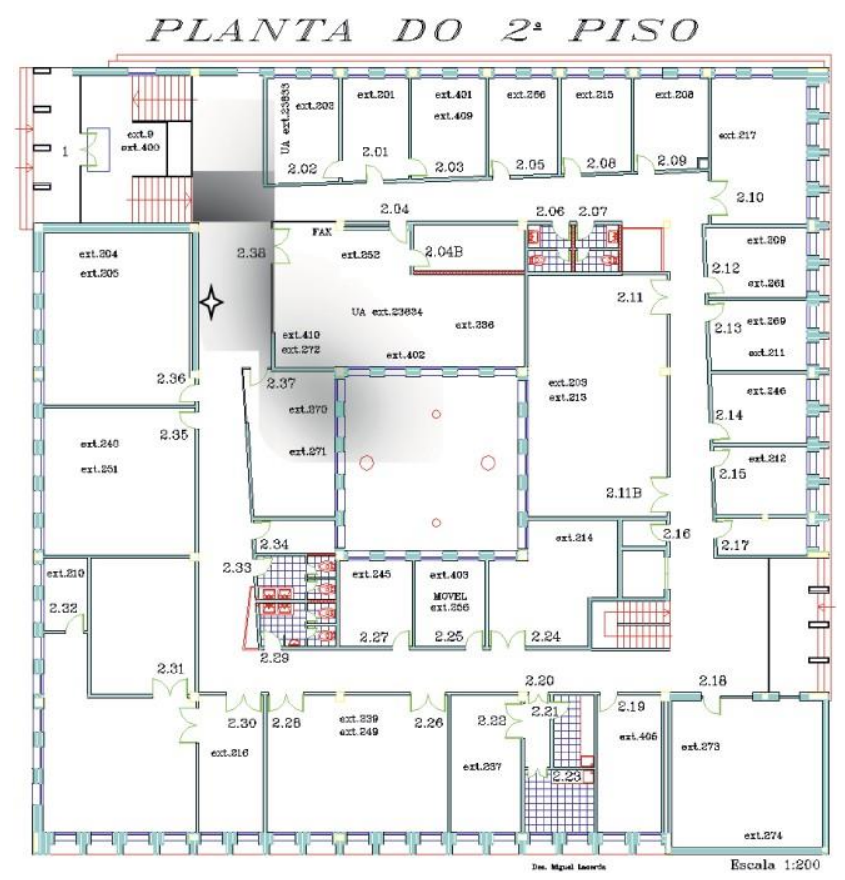

Fig. 17 Localization using the old localization map as an "a priori"

\subsection{A metric to tune up the localization system}

The metric proposed in Sect. 3.1 is here applied to the probability maps obtained by localization. In particular, Figs. 24, $25,26,27$ show the size of the "feasible location" areas, when the system considers respectively $1 \%$ and $0.5 \%$ of the best locations on the map. It is seen that "recycling" the old probability map gives a very good result in terms of the

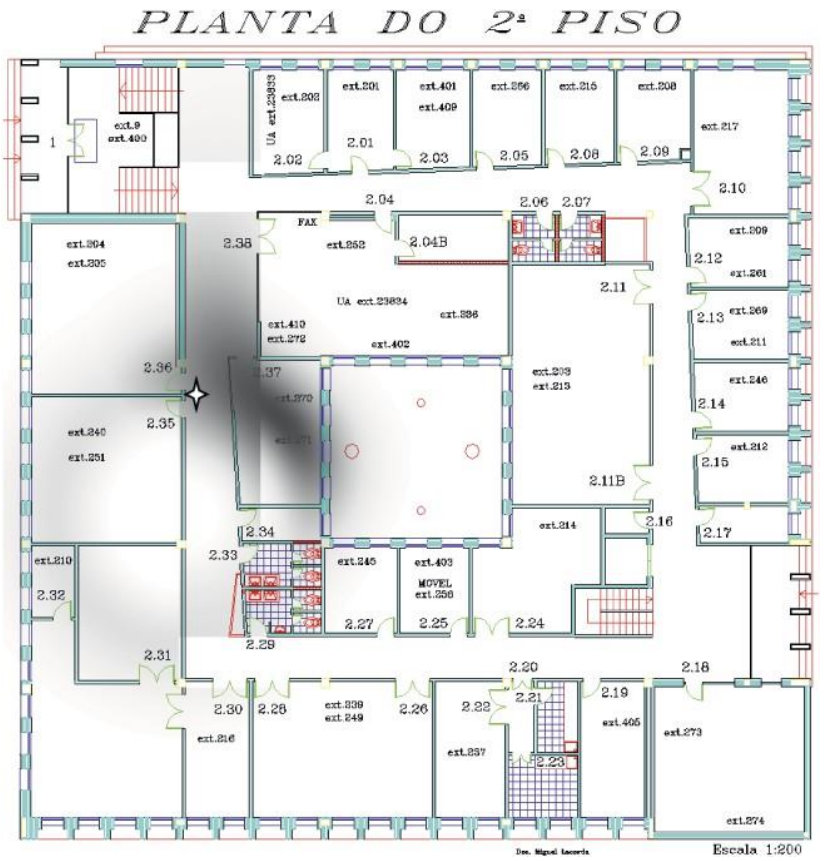

Fig. 18 Localization without recycling the old map as an "a priori"

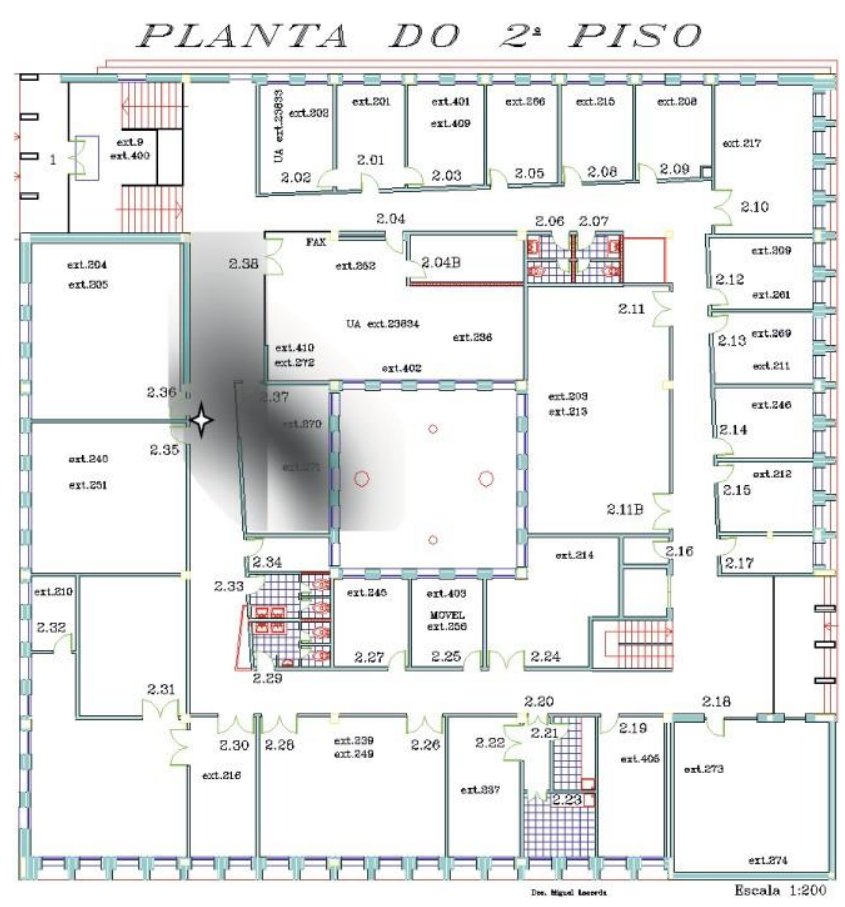

Fig. 19 Localization using the old localization map as an "a priori"

distance between the real location of the MT and the "feasible locations".

Figure 28 shows the results of an experiment involving applying the different thresholds (1\% and $0.5 \%)$ to the path analyzed in Sect. 4.3, both using and not using the old "a posteriori" map as the next "a priori". The figure shows the distance between the real location of the MT and 


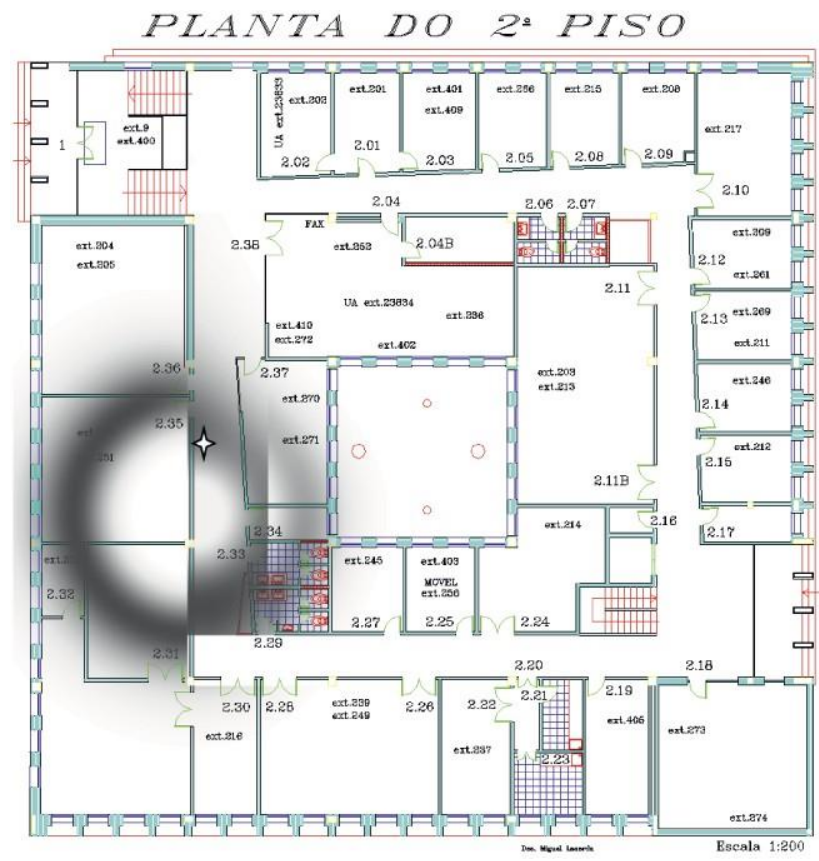

Fig. 20 Localization without recycling the old map as an "a priori"

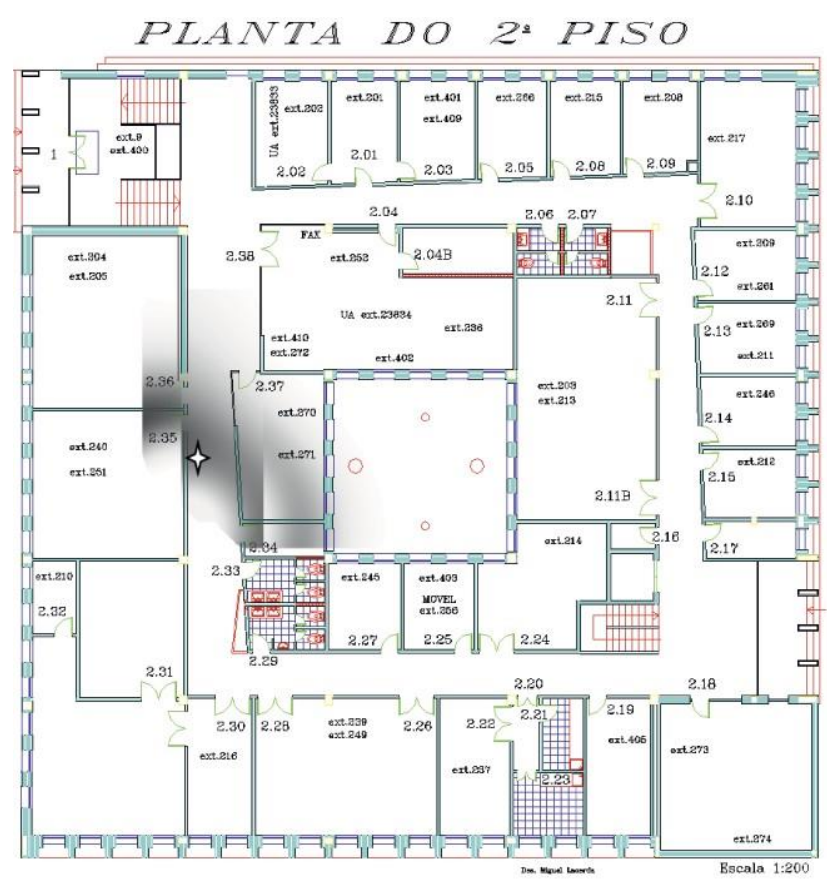

Fig. 21 Localization using the old localization map as an "a priori"

the "feasible locations" map against the step of localization. Each point represents a location on the map where we collected measurements about the signal strengths, we computed the probability maps using the different strategies, and we measured the distance between the real location and the closest point on the probability map. We collected 11 measurement for each strategy that was taken into account.

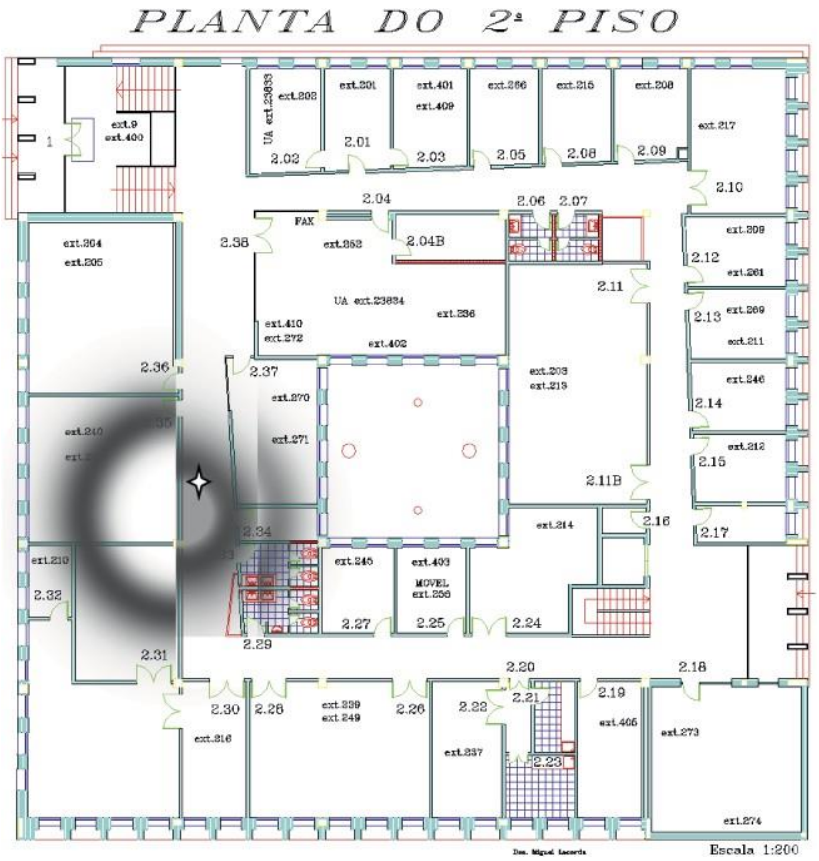

Fig. 22 Localization without recycling the old map as an "a priori"

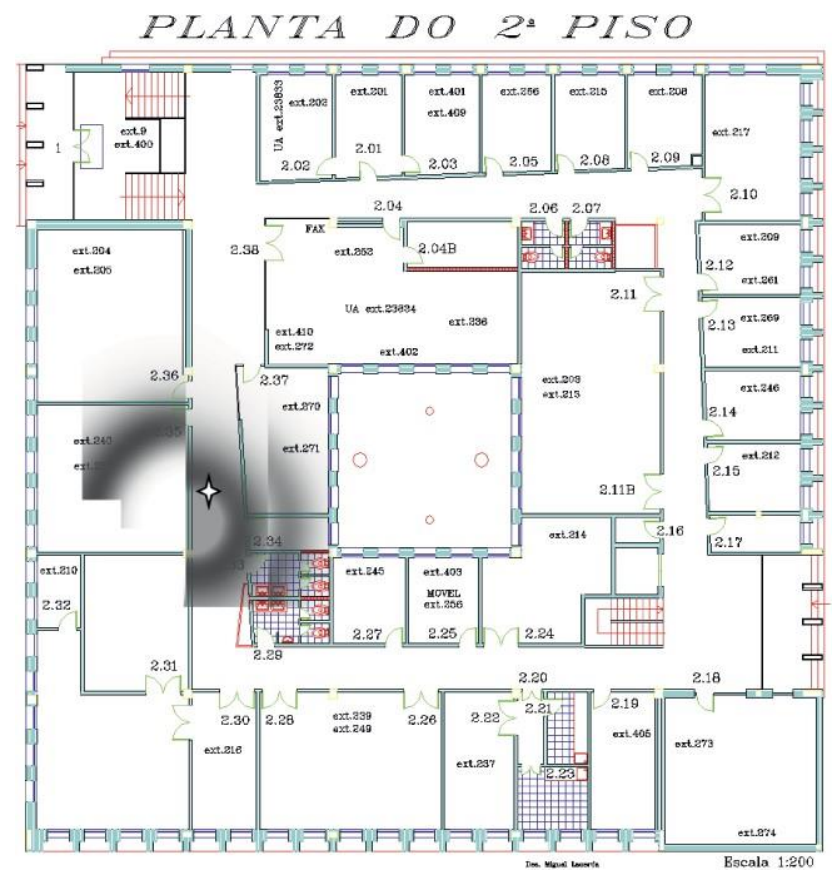

Fig. 23 Localization using the old localization map as an "a priori"

It is clear that there is a trade off between the size of the "feasible locations" map and the distance to the real location. The smaller the map is, the greater the precision of MT localization. On the other hand, it can be observed that in certain cases the real location of the MT is not inside the "feasible location" regions when the map is smaller, hence a lower threshold decreases the performance of the localization technique. 


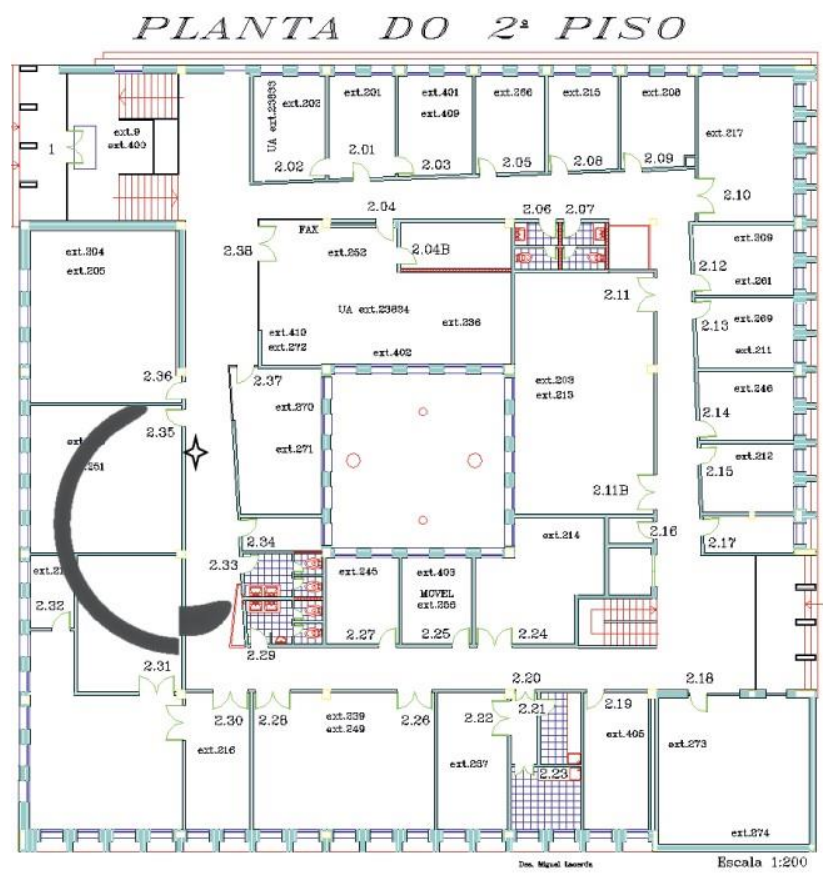

Fig. 24 Localization without recycling the old map, threshold at $1 \%$

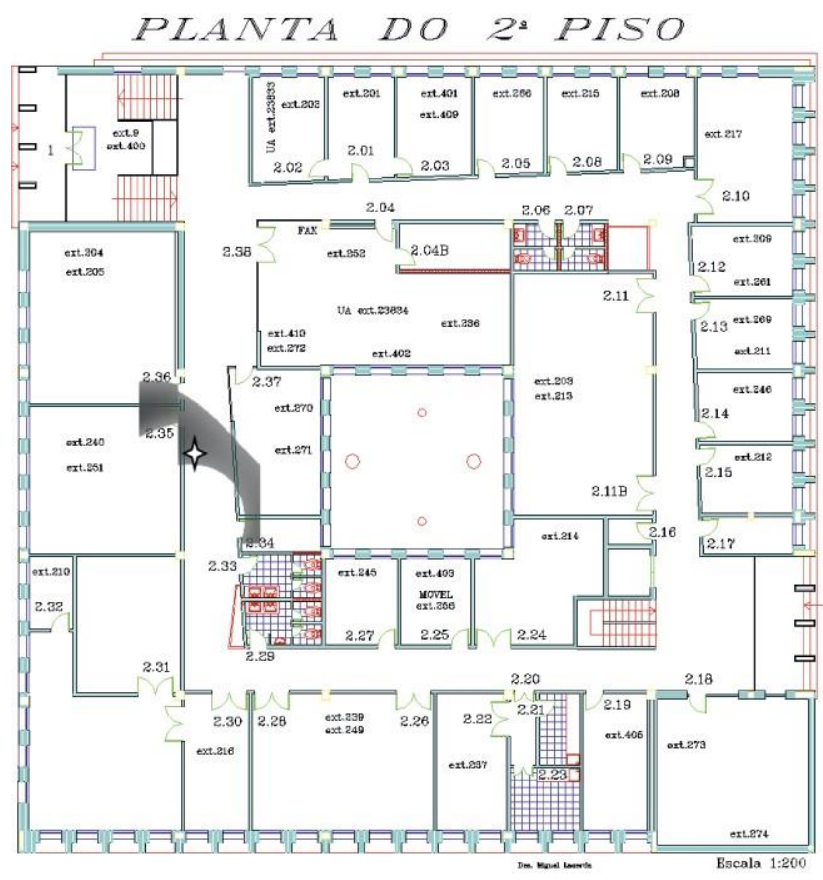

Fig. 25 Localization using the old localization map as an "a priori", threshold at $1 \%$

Thus, in a real deployment scenario, it will be necessary to consider the threshold for the "feasible locations" regions as a trade-off between precision and localization reliability.

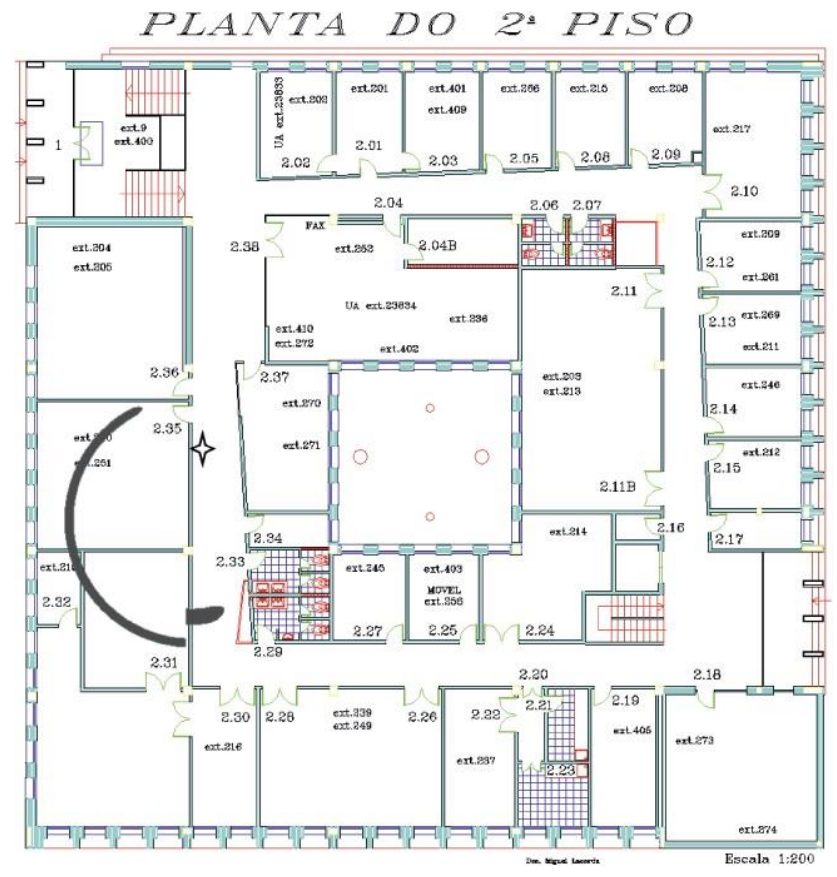

Fig. 26 Localization without recycling the old map, threshold at $0.5 \%$

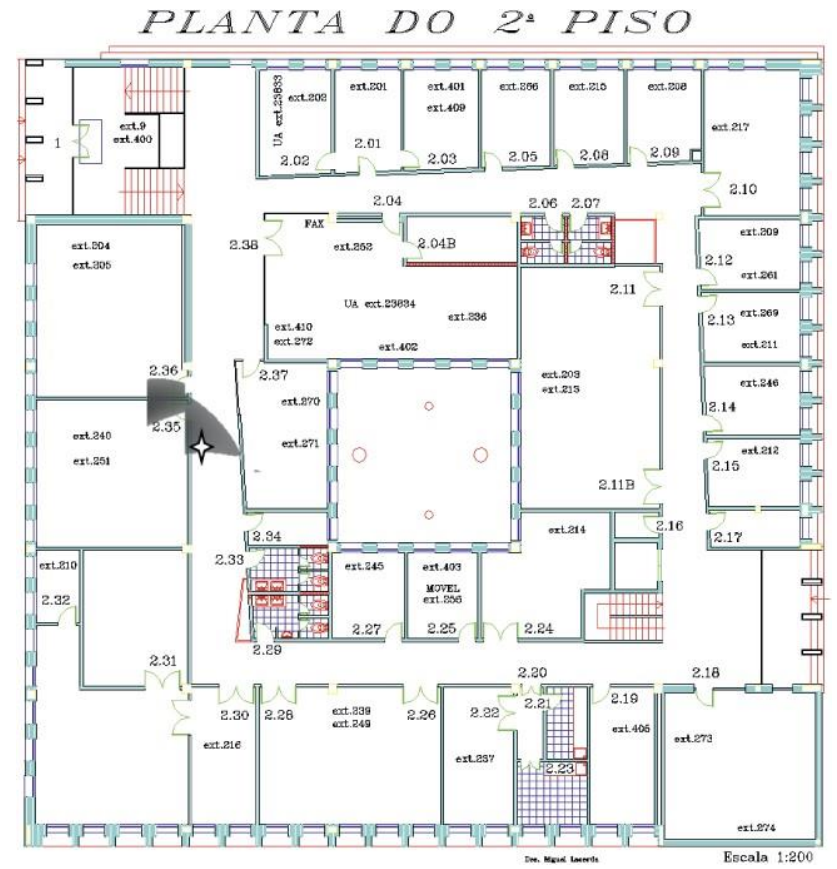

Fig. 27 Localization using the old localization map as an "a priori", threshold at $0.5 \%$

\section{Conclusions}

Indoor localization is still a challenging research topic. One way to improve the positioning procedure is to make use of all available environmental information. In this paper we have shown how negative information (information about where the mobile unit is not) can be incorporated into an 


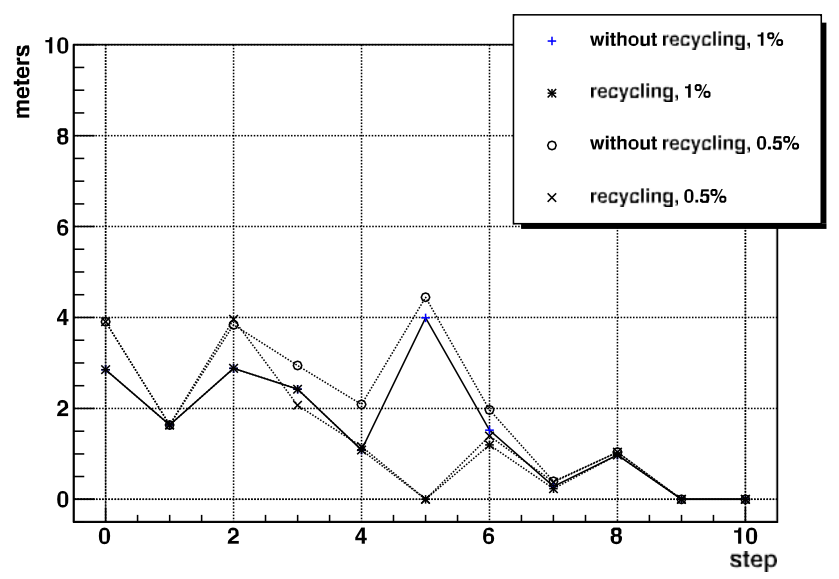

Fig. 28 Comparison of "recycling the a priori" algorithm against localizing without an "a priori", using the metric of Sect. 3.1

indoor positioning scenario. The paper showed the proof of concept for the proposed strategy, given that we applied a simple channel model, and assumed independent measurement errors. In addition, inspired by tracking algorithms, we extended our approach by exploiting the history of mobile terminal's location to assist the computation of the terminal location. The method based on probability map recycling outperformed the basic implementation.

For illustration purpose, we used a simple lognormal model without taking into account spatial correlation. However, correlated shadowing is shown to have significant impact on system performance in WLAN networks [36]. If a signal in a certain direction is attenuated by an obstruction, it is very likely that a received signal in close proximity is experiencing a similar shadowing effect. The assumption that shadowing losses are correlated among nearby links has been verified by experimental measurements [37]. Therefore is it important to improve statistical propagation models and include them in localization algorithms, what we intend to do in our future work.

Acknowledgements This work has been performed in the framework of the ICT project ICT-248894 WHERE2, which is partly funded by the European Union.

\section{References}

1. Want, R., Hopper, A., Falcao, V., \& Gibbons, J. (1992). The active badge location system. ACM Transactions on Information Systems, 10(1), 91-102.

2. Bahl, P., \& Padmanabhan, V. N. (2000). RADAR: an in-building RF-based user location and tracking system. In Proc. INFOCOM 2000, Tel Aviv, Israel, March 2000 (Vol. 2, pp. 775-784).

3. Irfan, N., Bolic, M., Yagoub, M., \& Narashiman, V. (2010). Localization of sensors in indoor environment with neural network method. Telecommunications Systems, 44(1), 149-158.

4. LaMarca, A., Chawathe, Y., Consolvo, S., et al. (2005). Place lab: device positioning using radio beacons in the wild. In Proc. IEEE international conference on pervasive computing and communications (Vol. 3468, pp. 116-133). Berlin: Springer.

5. De Luca, D., Mazzenga, F., Monti, C., \& Vari, M. (2006). Performance evaluation of indoor localization techniques based on RF power measurements from active or passive devices. EURASIP Journal on Applied Signal Processing, 2006(1), 1-11.

6. Priyantha, N. B., Chakraborty, A., \& Balakrishnan, H. (2000). The Cricket location-support system. In International conference on mobile computing and networking, Boston, Massachusetts, USA (pp. 32-43).

7. Han, G., Xu, H., Duong, T., Jiang, J., \& Hara, T. (2011). Localization algorithms of wireless sensor networks: a survey. Telecommunications Systems. doi:10.1007/s11235-011-9564-7. Preview published online on 4 August2011.

8. Wu, C., Sheng, W., \& Zhang, Y. (2006). Mobile self-localization using multi-dimensional scaling in robotic sensor networks. The International Journal of Intelligent Control and Systems, 11(3), $163-175$.

9. Costa, J., Patwari, N., \& Hero, A. (2006). Distributed weightedmultidimensional scaling for node localization in sensor networks. ACM Transactions on Sensor Networks, 2(1), 39-64.

10. Doherty, L., Pister, K. S. J., \& Ghaoui, L. E. (2001). Convex position estimation in wireless sensor networks. In Proc. of IEEE INFOCOM, April 2001 (Vol. 3, pp. 1655-1663).

11. Biswas, P., \& Ye, Y. (2004). Semidefinite programming for ad hoc wireless sensor network localization. In Proc. international conference on information processing in sensor networks (IPSN '04), Berkeley, CA, April 2004.

12. Savvides, A., Han, C., \& Srivastava, M. (2001). Dynamic finegrained localization in ad-hoc networks of sensors. In 7th ACM international conference on mobile computing and networking (Mobicom), Rome, Italy, July 2001 (pp. 166-179).

13. Aslindi, N., Pahlavan, K., Alavi, B., \& Li, X. (2006). A novel cooperative localization algorithm for indoor sensor networks. In IEEE international symposium on personal, indoor and mobile radio communications PIMRC '06, Helsinki, Finland (pp. 1-6).

14. Liu, J., \& Zhang, Y. (2008). Error control in distributed node selflocalization. EURASIP Journal on Advances in Signal Processing, January.

15. Ihler, A. T., Fisher, J. W., \& Moses, R. L. (2004). Nonparametric belief propagation for self-calibration in sensor networks. In Proceedings of the 3rd international symposium on information processing in sensor networks, Berkeley, CA, USA (pp. 225-233).

16. Savic, V., \& Zazo, S. (2009). Nonparametric boxed belief propagation for localization in wireless sensor networks. In Proceedings of IEEE SENSORCOMM, Athens, Greece, June 2009 (pp. 520525).

17. Savic, V., \& Zazo, S. (2009). Sensor localization using nonparametric generalized belief propagation in network with loops. In Proceedings of IEEE international conference on information fusion, Seattle, USA, July 2009 (pp. 1966-1973).

18. Wymeersch, H., Lien, J., \& Win, M. Z. (2009). Cooperative localization in wireless networks. Proceedings of the IEEE, 97(2). Special Issue on UWB Technology and Emerging Applications.

19. Dellaert, F., et al. (1999). Monte Carlo localization for mobile robots. In IEEE international conference on robotics and automation (ICRA), May 1999.

20. Hu, L., \& Evans, D. (2004). Localization for mobile sensor networks. In Proceedings of ACM MobiCom 2004, Philadelphia, USA, September 2004 (pp. 45-57).

21. Hoffmann, J., Spranger, M., \& Gohring, D. (2005). Making use of what you don't see: negative information in Markov localization. In Proc. of IEEE/RSJ international conference of intelligent robots and systems (IROS), Alberta, Canada, August 2005.

22. Koch, W. (2004). On negative information in tracking and sensor data fusion: discussion of selected examples. In Proc. of 7 th international conference on information fusion (pp. 91-98). 
23. Montemerlo, M., \& Thrun, S. (2003). Simultaneous localization and mapping with unknown data association using FastSLAM. In Proc. IEEE international conference on robotics and automation (pp. 1985-1991).

24. Baggio, A., \& Langendoen, K. (2008). Monte-Carlo localization for mobile wireless sensor networks. Ad Hoc Networks, 6(5), 718733.

25. Caceres, M. A., Sottile, F., \& Spirito, M. A. (2009). Adaptive location tracking by Kalman filters in wireless sensor networks. In Proc. of IEEE international conference on wireless and mobile computing, networking and communications.

26. Welch, G. F., \& Bishop, G. (2006). An introduction to the Kalman filter (Tech. Rep 95-041). University of North Carolina, Chapel

27. Sir Conan Doyle, A. (1890). The sign of thefour.

28. Rappaport, T. S. (2002). Wireless communications principles and practices. New York: Prentice-Hall.

29. Akl, R., Tummala, D., \& Li, X. (2006). Indoor propagation modeling at $2.4 \mathrm{GHz}$ for IEEE 802.11 networks. In Proc. 6th IASTED international multi-conference on wireless and optical communications, Banff.

30. Sadiki, T., \& Paimblanc, P. (2009). Modelling new indoor propagation models for WLAN based on empirical results. In Proc. UKSim 2009: 11th international conference on computer modelling and simulation, Cambridge, England, March 2009.

31. Stuedi, P., Chinellato, O., \& Alonso, G. (2005). Connectivity in the presence of shadowing in 802.11 ad hoc networks. In Proc. IEEE wireless communications and networking conference (WCNC), Mar. 2005 (pp. 2225-2230).

32. http://wifihopper.com/.

33. Goldsmith, A. (2005). Wireless communications, ISBN 9780521837163, September2005

34. Mathur, R., Klepal, M., McGibney, A., \& Pesch, D. (2004). Influence of people shadowing on bit error rate of IEEE 802.112.4 $\mathrm{GHz}$ channel. In 1st International symposium on wireless communication systems (ISWCS 2004), Port-Louis, Mauritius, September 2004 (pp. 448-452).

35. Chrysikos, T., Georgopoulos, G., \& Kotsopoulos, S. (2010). Impact of shadowing deviation on wireless channel characterization for a public indoor commercial topology at $2.4 \mathrm{GHz}$. In 2 nd International congress on ultra modern telecommunications (ICUMT 2010), Moscow, Russia, 18-20 October 2010 (pp. 18-20).

36. Butterworth, K. S., Sowerby, K. W., \& Williamson, A. G. (2000). Base station placement for in-building mobile communication systems to yield high capacity and efficiency. IEEE Transactions on Communications, 48(4), 658-669.

37. Agrawal, P., \& Patwari, N. (2009). Correlated link shadow fading in multi-hop wireless networks. IEEE Transactions on Wireless Communications, 8(8), 4024-4036. 